\title{
Action du calcaire sur la germination et la croissance du chêne rouge d'Amérique ( $Q$. rubra $L$.)
}

\author{
J. GELPE, J. TIMBAL \\ avec la collaboration technique de G. COURRIER et Ph. ROSSETTO \\ INRA, Département des Recherches forestières \\ Laboratoire de Sylviculture et Ecologie, Domaine de l'Hermitage \\ Pierroton, F 33610 Cestas
}

\section{Introduction}

La forte croissance juvénile du chêne rouge d'Amérique (Quercus rubra L. $=Q$. borealis Michx) fait que cette essence est de plus en plus utilisée pour le reboisement en France et en Europe. Cependant, son utilisation forestière est limitée par son caractère calcifuge, mais il n'existe pas de données précises sur ce point, car à notre connaissance, très peu de travaux ont été effectués sur ce sujet.

Kostov (1969) a cultivé du chêne rouge sur un tchernozem de pH 7.4 contenant 4,82 p. 100 de calcaire : dans ces conditions la survie et la croissance étaient réduites. Après culture sur différents types de tchernozems, un optimum de croissance est observé à $\mathrm{pH}$ 6,7-6,8 avec un effet dépressif des bas $\mathrm{pH}(\mathrm{pH} \mathrm{5,4)}$ et surtout des forts pH (pH 8,4) (Kostov, 1975). Très récemment, Devildoer (1984) a confirmé par des expériences sur racines excisées le caractère calcifuge de cette espèce.

La germination et la culture de jeunes plants dans des sols représentatifs des conditions édaphiques d'une bonne partie du Sud-Ouest de la France, a permis d'apprécier l'effet de cette calcifugie sur la germination et la croissance.

\section{Matériel et méthode}

L'expérience a consisté à faire germer, en serre, dans des pots de culture de 51 , des glands de chêne rouge de deux origines connues, et à suivre leur croissance durant toute une saison de végétation. Le substrat de culture est un limon acide prélevé en forêt (voir annexe 1). Cinq doses de calcaire, sous forme de $\mathrm{CaCO}_{3}$ pulvérulent, ont été testées : 0 p. 100 (témoin), 1 p. 100,2 p. 100,5 p. 100 , et 10 p. 100 du poids sec de terre. Chaque traitement des deux origines, a été répété 25 fois, ce qui fait un total de 250 pots répartis en 10 blocs. 
Les semis ont été faits au mois de mai 1984. L'expérience a été arrêtée en mai 1985, couvrant ainsi une saison entière de végétation et permettant ainsi de calculer le taux de survie à la fin de celle-ci par la présence ou l'absence de débourrement. Durant l'expérience tous les pots ont été régulièrement arrosés afin de maintenir la terre à la capacité au champ (annexe 2). Aucune fertilisation n'a été apportée.

\section{Résultats}

\subsection{Phénologie}

La germination des glands s'est déroulée d'une façon normale et identique dans toutes les modalités. Ce n'est qu'au moment de la deuxième pousse que des différences sont apparues, révélant une réponse de type «tout ou rien» au calcaire. En effet, alors que les témoins présentaient des pousses de 10 à $20 \mathrm{~cm}$ de longueur et de grandes feuilles vertes, tous les autres traitements (de 1 à $10 \mathrm{p} .100 \mathrm{de} \mathrm{CaCO}_{3}$ ) présentaient des allongements moindres, voire nuls, et surtout, souvent, des feuilles ayant tous les symptomes d'une chlorose : limbe de surface réduit, surtout en largeur, et de couleur vert-jaune, souvent en marbrures le long des nervures. A l'automne 1984 , la coloration rouge-brun des feuillos, si caractéristique de l'espèce, est apparue en moyenne plus tôt dans les modalités avec calcaire que sur les témoins.

\subsection{Taux de survie}

Les différences entre les taux de mortalité des plants témoins et des plants cultivés en présence de calcaire est importante et significative. Par contre, il n'y a pas de différence significative entre les différents traitements avec calcaire, ce qui est aussi une réponse de type « lout ou rien » à la présence de calcaire.

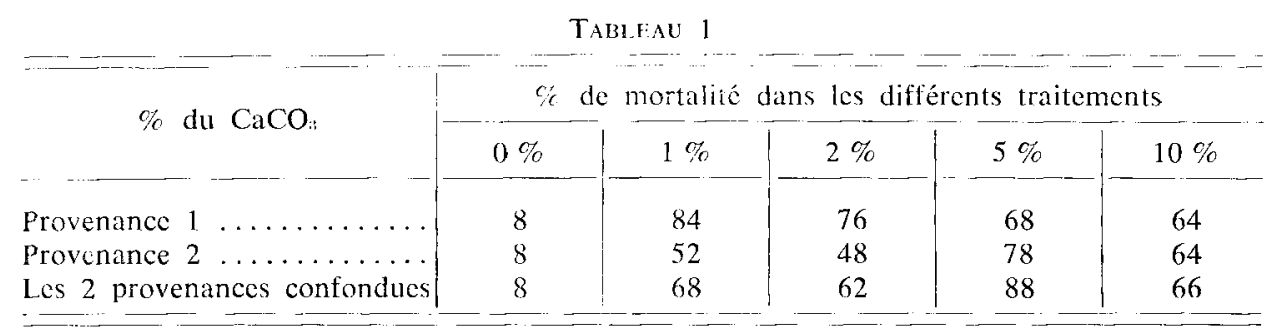

\subsection{Croissance}

Un effet de type «tout ou rien" apparaît encore. En effet, il y a une différence très significative (à 1 p. 100) entre les témoins et les traitements mais aucune différence significative entre ces derniers.

Il n'y a pas non plus de différences significatives entre les deux provenances testées ni d'interaction entre l'origine et le traitement. 


\section{TABLEAU 2}

Croissance en hateur.

\begin{tabular}{|c|c|c|c|c|}
\hline \multicolumn{5}{|c|}{ Hauteur totale moyenne $(\mathrm{en} \mathrm{mm})$ selon les traitements } \\
\hline & & Prov. I & Prov. II & Les 2 prov. confondues \\
\hline $0 \%$ & & 171,88 & 187,67 & 176,78 \\
\hline $1 \%$ & $\ldots \ldots \ldots \ldots$ & 136,72 & 136,36 & 136,54 \\
\hline $2 \%$ & $\ldots \ldots \ldots \ldots$ & 140,44 & 147,28 & 143,86 \\
\hline $5 \%$ & $\ldots \ldots \ldots \ldots$ & 129,20 & 142,40 & 132,50 \\
\hline $10 \%$ & $\ldots \ldots \ldots$ & 132,71 & 129,52 & 131,08 \\
\hline
\end{tabular}
partielles.

Il en est de même si on considère, non plus la pousse totale, mais les pousses

\subsection{Croissance racinaire}

Les systèmes racinaires des plants témoins se caractérisent par l'abondance des fines racines alors que, quel que soit le traitement, les plants sur calcaire ont un gros pivot (parfois même plus gros en diamètre que la tige aérienne) et très peu dé fines racines.

Tous les plants sont apparus mycorhizés par Cenococum graniforme, avec des taux semblables selon la provenance et le traitement.

En ce qui concerne les biomasses racinaires les résultats sont tout à fait semblables à ceux obtenus sur la croissance aérienne.

\subsection{Polycyclisme}

Bien que le dispositif expérimental n'ait pas été réalisé dans le but d'étudier le polycyclisme chez le chêne rouge, il apporte cependant des données intéressantes sur ce point. Selon les individus, de 1 à 3 pousses ont été faites au cours de la saison de végétation. Mais ce taux de polycyclisme apparaît comme relativement indépendant de la provenance et du traitement. De plus, il apparaît clairement que, en moyenne, les individus polycycliques présentent une croissance supérieure en hauteur à celle des monocycliques.

\section{Conclusion}

Cette expérience met en évidence la sensibilité du chêne rouge au calcaire : celle-ci apparaît avec 1 p. 100 de $\mathrm{CaCO}_{3}$ dans le substrat. Cette sensibilité se manifeste par un faible développement racinaire (surtout de fines racines), une plus faible croissance aérienne, et surtout un fort taux de mortalité dès la première année de végétation. Par contre, le processus de germination n'est pas affecté.

Des doses de calcaire inférieures à 1 p. 100 n'ont pu être utilisées à cause des difficultés d'homogénéisation du mélange du calcaire et du substrat, ce qui n'a pas permis de définir un éventuel seuil de sensibilité, ce qui pourrait sans doute être fait en solution nutritive. 
De même, ce type d'expérience ne peut donner d'indications sur la réaction du chêne rouge (à ce stade juvénile ou à un âge plus avancé) dans un sol carbonaté en profondeur mais décarbonaté en surface, décalcifié ou non; or ce cas est fréquent dans la nature.

Il n'y a pratiquement pas de différence entre les deux provenances testées, bien que l'une (La Houve) apparaisse un peu moins sensible que l'autre.

Parallèlement aux observations sur la sensibilité au calcaire, le chêne rouge apparaît comme une espèce polycyclique (majorité d'individus à 2 cycles). Ce polycyclisme apporte un gain de croissance mais est peut-être une des causes des problèmes de fourchaison constatés chez cette espèce.

\author{
Reçu le $1^{\mathrm{er}}$ juillet 1985. \\ Accepté le 24 octobre 1985.
}

\author{
Summary \\ Limestone action on germination and early growth of \\ northern red oak (Quercus rubra L.)
}

\begin{abstract}
Limestone sensitivity of Quercus rubra suggested by its bad growth on carbonated chernozems (Kostov, 1969, 1975), and demonstrated by an experiment on excised roots (Devilder, 1984), has been studied in forest soil conditions of South-western France.

The experiment consisted of raising seedlings in an acid desaturated loam taken form a forest soil. Pulverulent $\mathrm{CaCO}_{3}$ was also added to the medium at differents rates : 0 p. 100, 1 p. 100,2 p. 100 and 10 p. 100 of dry weight. The material consisted of two provenances. Each was represented by 25 seelings. Several attribues were measured after germination at the end of the first growing season : phenology, survival, height growth and polycyclism rates.

After the germination stage, which was the same in each treatment, differences in survival became significant : in the 1 p. 100 treatment, mortality and growth reduction were already important, and leaf chlorosis symptoms were noticeable. Root biomass was similarly significant (very few small roots in $\mathrm{CaCO}$ : treatments). Also, in autumn, leaf fall occurred carlier in $\mathrm{CaCO}_{3}$ treatments. There was no significant difference between the two provenances tested. Height growth followed a polycyclic pattern; polycyclic seedlings having a better height growth but also, perhaps, more forking afterwards.
\end{abstract}

Key words : Red oak (Quercus rubra), limestone sensitivity, chlorosis, seedling growth, polycyclism.

\title{
Références bibliographiques
}

DeVILDER R., 1984. Quelques aspects anatomiques et physiologiques de la racine primaire du chêne rouge d'Amérique (Q. rubra L.). Mémoire de D.E.A., Université de Bordeaux III, laboratoire de biologie et physiologie végétale, $79 \mathrm{p}$.

Keller et al., 1980. Le chêne rouge (Q. rubra L.) en France. Rev. For. Fr., 5, 419-451.

Kostov K.D., 1969. Growth of Quercus borealis on carbonate chernozem. Gorskostop Nauka, Sofija, 6 (1), 13-21 (10 refs, bulg., russe, angl., 7 fig., 5 tbl.).

Kostov K.D., 1975. Growth of Quercus rubra on soils differing in acidity. Gorskostop Nauka, 1975, 12 (5), 49-60 (bulg., russe, angl., 10 refs). 


\section{Annexe 1 : Substrat de culture}

Horizon A 1 d'un sol limon acide prélevé en forêt du Mas Agenais (47).

Caractéristiques physico-chimiques (valeurs moyennes sur 11 échantillons) :

- $\mathrm{pH}$ (eau) $\ldots \ldots \ldots \ldots \ldots \ldots \ldots .4,2$ p. 100

— limons fins ............ 30,0 p. 100

- limons grossiers .......... 31,6 p. 100

- argile $\ldots \ldots \ldots \ldots \ldots \ldots \ldots \ldots, 14,8$ p. 100

$-\mathrm{CaO} \ldots \ldots \ldots \ldots \ldots \ldots \ldots \ldots . .0,3$ p. 100

\section{Annexe 2 : Conditions de $\mathrm{pH}$}

pH de l'eau d'arrosage (eau du réseau) : 7,2 à 7,5.

Evolution du pH des substrats.

\begin{tabular}{|c|c|c|}
\hline Dose de calcaire & pH au début de l'expérience & pH à la fin de l'expérience \\
\hline 0 p. 100 & 5,4 & 5,0 \\
\hline 1 p. $100 \ldots \ldots \ldots \ldots \ldots \ldots$ & 6.9 & 7,7 \\
\hline$\ldots \ldots \ldots \ldots \ldots \ldots$ & 7,1 & 7,8 \\
\hline 5 p. $100 \ldots \ldots \ldots \ldots \ldots \ldots$ & 7,3 & 7,9 \\
\hline$\ldots \ldots \ldots \ldots \ldots \ldots$ & 7,4 & 8,0 \\
\hline
\end{tabular}

L'augmentation du pH dans les traitements avec calcaire suggère une libération progressive du calcaire actif dans la solution du sol. 